\title{
Targeted protein depletion in Saccharomyces cerevisiae by activation of a bidirectional degron
}

\author{
Marc Jungbluth, Christian Renicke, Christof Taxis
}

\begin{abstract}
Background: Tools for in vivo manipulation of protein abundance or activity are highly beneficial for life science research. Protein stability can be efficiently controlled by conditional degrons, which induce target protein degradation at restrictive conditions.

Results: We used the yeast Saccharomyces cerevisiae for development of a conditional, bidirectional degron to control protein stability, which can be fused to the target protein N-terminally, C-terminally or placed internally. Activation of the degron is achieved by cleavage with the tobacco etch virus (TEV) protease, resulting in quick proteolysis of the target protein. We found similar degradation rates of soluble substrates using destabilization by the $\mathrm{N}$ - or C-degron. C-terminal tagging of essential yeast proteins with the bidirectional degron resulted in deletion-like phenotypes at non-permissive conditions. Developmental process-specific mutants were created by $\mathrm{N}$ - or C-terminal tagging of essential proteins with the bidirectional degron in combination with sporulationspecific production of the TEV protease.

Conclusions: We developed a system to influence protein abundance and activity genetically, which can be used to create conditional mutants, to regulate the fate of single protein domains or to design artificial regulatory circuits. Thus, this method enhances the toolbox to manipulate proteins in systems biology approaches considerably.
\end{abstract}

\section{Background}

One of the basic experiments in cell biology is to remove a specific protein, conduct a phenotypic analysis and from that deduce the function of the protein. Often, the main problem is to remove the protein of interest quickly and efficiently. Many techniques have been developed to disturb the synthesis, activity or abundance of a selected protein [1-7]. The addition of a destabilizing sequence (degron) is used to reduce the half-life of the target protein by inducing proteasomal proteolysis [8]. A recent development to target proteins for degradation is the auxin-inducible degron, a relative of the PROTACS system. Both methods use chemical compounds to tether a protein to an ubiquitin-protein-ligase, thereby inducing polyubiquitylation and degradation of the target protein $[9,10]$. Other methods utilize small chemical compounds to change the destabilization activity of protein domains

\footnotetext{
* Correspondence: taxis@staff.uni-marburg.de
Department of Genetics, Philipps-Universität Marburg, Karl-von-Frisch-Str. 8,

*Correspondence: taxis@staff.uni-marburg.de
Department of Genetics, Philipps-Universität Marburg, Karl-von-Frisch-Str. 8, 35043 Marburg, Germany
}

(c) 2010 Jungbluth et al; licensee BioMed Central Ltd. This is an Open Access article distributed under the terms of the Creative Commons Attribution License (http://creativecommons.org/licenses/by/2.0), which permits unrestricted use, distribution, and reproduction in any medium, provided the original work is properly cited.
$[11,12]$, or degrade target proteins employing the bacterial protease ClpXP [13].

One very widespread method is the release of an $\mathrm{N}$-degron by proteolysis applying the ubiquitin-fusion technique. The fusion of ubiquitin to the N-terminus of a protein results in cleavage by deubiquitylating enzymes between ubiquitin and the protein. Thereby, almost all amino acids can be exposed at the N-terminus of a protein. The technique led to the formulation of the N-end rule, which states that the in vivo half-life of a protein is related to the identity of the $\mathrm{N}$-terminal amino acid [14-16]. In yeast 12 amino acids (D, E, F, H, I, K, L, N, $\mathrm{Q}, \mathrm{R}, \mathrm{W}$, and $\mathrm{Y}$ ) destabilize a protein if exposed at the $\mathrm{N}$-terminus. Eight of these destabilizing amino acids are directly recognized by the ubiquitin-protein-ligase Ubr1 (primary destabilizing residues), whereas four have to be modified by deamidation and/or arginylation (tertiary and secondary destabilizing residues) before. Recognition by Ubr1 leads to polyubiquitylation of the protein followed by proteasomal proteolysis $[17,18]$. Varshavsky and his co-workers developed a temperature-sensitive 
degron for the creation of conditional mutants [19]. This technique has been used effectively to investigate functions of many essential proteins in Saccharomyces cerevisiae, although it is often necessary to use high level expression of $U B R 1$ to induce a temperature-sensitive phenotype [20-24].

Recently, the tobacco etch virus (TEV) protease induced protein instability (TIPI) system was developed as another tool to create conditional mutants. A degradation tag (TDegF) with a dormant $\mathrm{N}$-degron is fused to the N-terminus of a target protein. Presence of the TEV protease leads to site-specific proteolysis of the tag and exposure of the $\mathrm{N}$-degron. This initiates destabilization of the target protein. Fast and efficient cleavage of the substrate is achieved utilizing a TEV protease (named $\mathrm{pTEV}^{+}$protease) with enhanced processivity towards the TDegF tag. Thus, by regulation of $p T E V^{+}$protease expression the TIPI system allows to control target protein abundance [25].

One of the well studied degrons, which might be useful for protein depletion, is the C-terminal degron of the mouse ornithine decarboxylase (cODC) which is conserved in vertebrates [26]. It induces rapid, proteasomal proteolysis independent of polyubiquitylation [27]. The half-life of full-length mouse ornithine decarboxylase expressed in yeast has been found to be 10 minutes [28]. Two elements within the degron mediate the destabilization activity. The first is a cysteine-alanine motif, which is important for proteasomal association. The second consists of an unstructured region flanking the cysteine-alanine motif, comprising 16 amino acids upstream and 19 downstream [26,29]. The cODC degron destabilizes a protein only if present at the very C-terminus [30]. Moving the cysteine-alanine motif only a few amino acids closer to the C-terminus or farther away impairs the destabilizing activity [26]. The cODC degron has been used to destabilize proteins in the fungus Saccharomyces cerevisiae [28], the plant Nicotiana tabacum cv. Xanthi [31], mammalian cell culture [32] or to study protein degradation in vivo and in vitro [33,34], though no conditional version of the degron has been developed. We reasoned that it should be possible to create a conditional C-degron by constructing a tag with the cODC degron kept inactive due to a protective peptide added C-terminally to the cODC degron. Activation of the tag is achieved by site-specific proteolysis resulting in activation of the cryptic C-degron. We chose a dormant N-degron [25] as protective group. Thus, we created a bidirectional degron consisting of two degrons, which inactivate each other until TEV protease cleavage. This bidirectional degron can be fused to the target $\mathrm{N}$-terminally, C-terminally or placed internally to regulate protein abundance in vivo.

\section{Results and Discussion}

\section{Construction of conditional C-degrons}

The structure of the bidirectional degrons is shown in Figure 1A. We chose the tobacco etch virus (TEV) protease to activate the $\mathrm{C}$-degrons due to our previous experience with the development of the TEV protease induced protein instability (TIPI) system [25]. The degrons are based on the conditional N-degron TDegF, which consists of an unstructured spacer region, the TEV protease cleavage site ENLYFQ-F, an N-degron and an affinity domain derived from the human protein SF3b155 (amino acids 381 to 424). This affinity domain binds strongly to the human protein p14, which is fused to the TEV protease, thereby enhancing processivity of the protease towards the TDegF tag [25]. The bidirectional degrons were created by insertion of sequences containing the cysteine-alanine motif and surrounding amino acids (8 or 14) of the C-terminal mouse ornithine decarboxylase degron (cODC) into the unstructured spacer region in front of the TEV protease recognition site. In both constructs, the cysteine-alanine motif was placed 19 amino acids upstream of the cleavage site. Thus, the newly developed bidirectional degrons consist of a C-degron separated from an $\mathrm{N}$-degron by the TEV protease recognition site (Figure 1A). Due to this arrangement, both degrons are kept inactive until the tag is cleaved by the TEV protease. The usage of the bidirectional degron for conditional destabilization of target proteins is illustrated in Figure 1B: The GFPcODC1-TDegF-RFP tag is fused to the 3'- end of the target gene. Expression of the $p T E V^{+}$protease gene is controlled by an appropriate promoter (inducible, active during a specific cell-cycle stage or developmental process). Upon expression of the $p T E V^{+}$protease, the tag is cleaved at the consensus site (ENLYFQ-F) if it is exposed to the cytoplasm or the nucleus. This cleavage leads to activation of the two dormant degrons resulting in rapid proteasomal proteolysis of the target protein and the RFP. The sequences of the N-degron TDegF as well as the bidirectional degrons CODC1-TDegF and cODC2-TDegF are given in Figure 1C.

Due to the modular design of our constructs, the TEV protease cleavage site might be flanked by stabilizing or destabilizing sequences. Depending on the modules used, internal tagging of a two domain protein followed by in vivo proteolytic cleavage leads to destabilization of the whole protein, destabilization of one domain or separation of the domains (Figure 1D). Using this strategy, the TIPI system is expanded not only by a C-degron, but gains the possibility to control the fate of protein domains after separation. The features of the newly developed constructs are summarized in Table 1. 


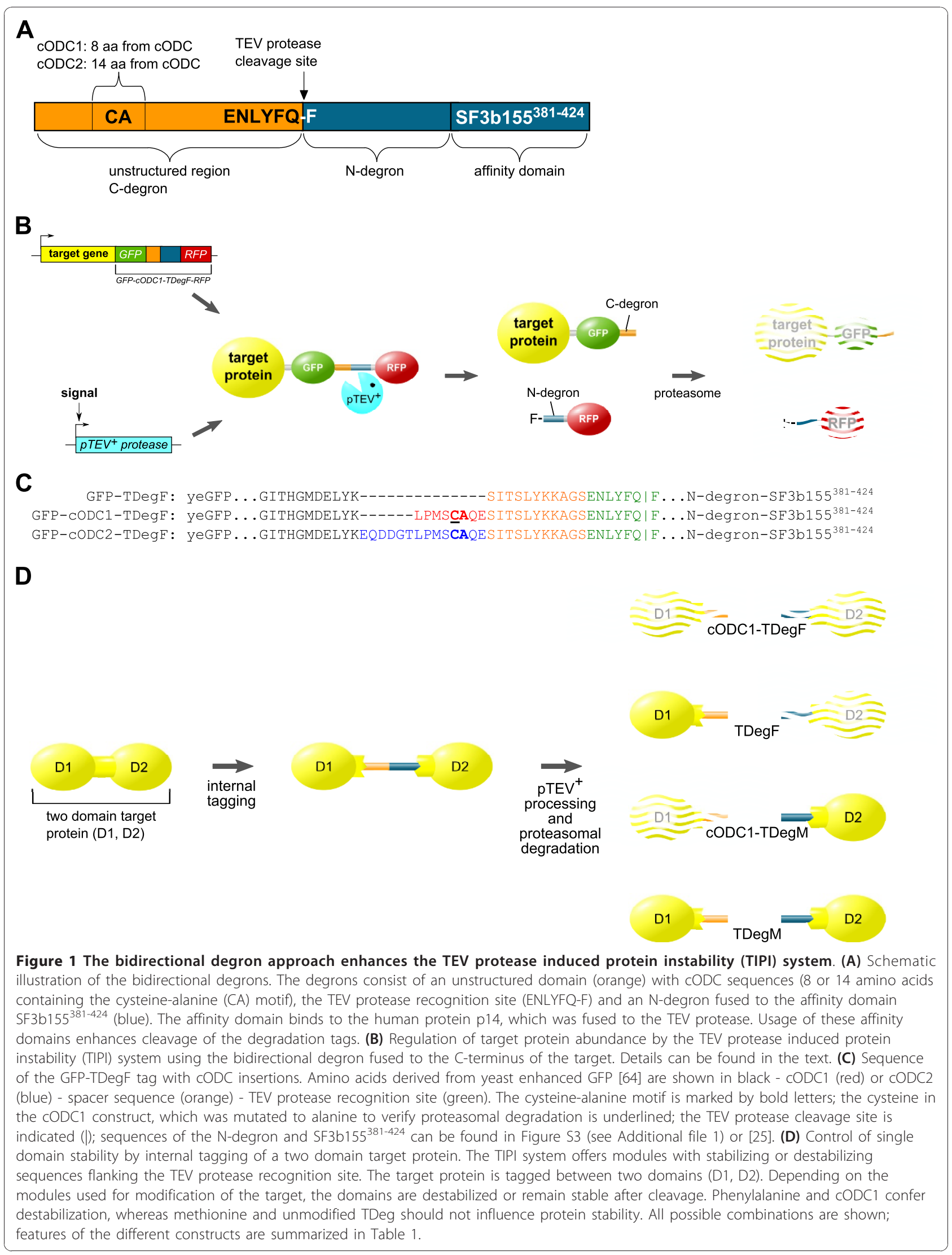


Table 1 Tags for protein degradation or cleavage

\begin{tabular}{lcccc}
\hline Constructs & $\begin{array}{c}\mathbf{N} \text { - } \\
\text { degron }\end{array}$ & $\begin{array}{c}\text { C- } \\
\text { degron }\end{array}$ & $\begin{array}{c}\text { Bidirectional } \\
\text { degron }\end{array}$ & Source \\
\hline TDegF & + & - & - & {$[25]$} \\
\hline TDegM & - & - & - & {$[25]$} \\
\hline GFP-CODC1-TDegF- & + & + & + & $\begin{array}{c}\text { This } \\
\text { Work }\end{array}$ \\
RFP & + & + & + & $\begin{array}{c}\text { This } \\
\text { Work }\end{array}$ \\
\hline GFP-CODC1-TDegF & + & + & - & $\begin{array}{c}\text { This } \\
\text { Work }\end{array}$ \\
\hline CODC1-TDegM & - & + & + & $\begin{array}{c}\text { This } \\
\text { work }\end{array}$ \\
\hline CODC2-TDegF & + & + & & +
\end{tabular}

The sequence of TDegM is similar to TDegF with the exception of the TEV protease cleavage site (ENLYFQ-M replaces ENLYFQ-F). The sequences for CODC1-TDegF and CODC2-TDegF differ in the length of the CODC sequence inserted into TDegF (Figure 1A, C). The ability to act as an N-degron, a Cdegron or a bidirectional degron is indicated for all constructs (+).

\section{Rapid protein depletion induced by the cODC degron}

We fused two different bidirectional degrons (cODC1/2TDegF) between the green fluorescent protein (GFP) and the red fluorescent protein mKate (RFP) to obtain tester proteins. The behavior of the tester proteins was assessed upon $p T E V^{+}$protease [25] expression. We observed complete cleavage of the tester proteins, depletion of the F-RFP as well as cODC-dependent destabilization of the GFP. Interestingly, we observed complete disappearance of the GFP signal only in case of the cODC1 construct (Figure 2A), showing that cODC1 mediates stronger destabilizing activity than cODC2.

For further characterization of the newly created C-degrons, we fused GFP-cODC1-TDegF-RFP and GFPcODC2-TDegF-RFP to the C-terminus of Cap2. Both constructs localized to patches near the plasma membrane, mostly within the bud. This localization pattern was found for Cap2-GFP as well [35], showing that addition of the tags did not influence subcellular localization. We followed the GFP- and RFP-fluorescence by live-cell imaging in presence and absence of the $\mathrm{pTEV}^{+}$ protease. Both, GFP- and RFP-fluorescence decreased in presence of the protease, almost complete loss of green fluorescence was observed in cells containing Cap2GFP-cODC1-TDegF-RFP (Figure 2B). This demonstrates that membrane association of target proteins does not hamper proteolytic cleavage and depletion. A similar behavior has been found for substrates modified with the TDegF tag as well [25]. The cODC1-TDegF tag was constructed as a conditional degron. To clarify, whether the cODC sequences exert destabilizing activity on a protein without $\mathrm{pTEV}^{+}$protease cleavage, we assessed the stability of the tester proteins GFP-TDegF-RFP, GFP-cODC1-TDegF-RFP, GFP-cODC2-TDegF-RFP and GFP-cODC1-TDegF (a shortened construct lacking the RFP) after inhibition of protein synthesis. To do so, the cells were treated with the translation elongation inhibitor cycloheximide at the same time as production of the $\mathrm{pTEV}^{+}$protease was induced. As expected, the tester proteins were not processed due to absence of the protease. We observed no degradation of the tester proteins GFP-TDegF-RFP, GFP-cODC1-TDegF-RFP, and GFPcODC2-TDegF-RFP, showing that the cODC sequences we used do not destabilize these constructs without activation of the degradation tag. However, no signal of the GFP-cODC1-TDegF construct was detected after cycloheximide treatment. Moreover, the steady-state level of this protein was reduced compared to the other constructs (Figure 2C). This indicates that the construct is destabilized without TEV protease cleavage. Presumably, this destabilization is induced by the largely unstructured SF3b155 $5^{381-424}$-domain [36]. It is known that an unstructured domain serves as a weak signal for proteolysis by the ubiquitin proteasome system $[37,38]$. Although the SF3b155 $181-424$-domain is present in all our tester proteins, destabilization occurs only in case of the GFP-cODC1-TDegF construct, which lacks a folded domain at the $\mathrm{C}$-terminus of the protein.

Mouse ODC is degraded by the proteasome independently of the ubiquitin system [27]. Therefore, reduced proteasomal activity should lead to incomplete proteolysis of a cODC1-containing tester construct. Using the cyan fluorescent protein (CFP) containing tester construct CFP-cODC1-TDegF-RFP, we observed that $p T E V$ + protease expression led to accumulation of cleavage intermediates in pre1-1 pre2-2 or pre1-1 pre4-1 mutant cells $[39,40]$ indicating incomplete proteolysis of CFPcODC1-TDeg and F-RFP fragments (Figure 2D). Proteasomal degradation of mouse ODC depends on the cysteine-alanine motif, which is necessary for proteasomal association of the degron [26,29]. Mutation of this motif should abolish rapid depletion of cleaved GFPcODC1-TDegF-RFP. We replaced the cysteine by an alanine and compared the behavior of this construct with GFP-cODC1-TDegF-RFP. As expected, we found complete depletion of GFP-cODC1-TDeg after $\mathrm{pTEV}^{+}$ protease cleavage, whereas GFP-cODC $1^{\mathrm{C} 243 \mathrm{~A}}$-TDeg was much less prone to degradation (Figure 2E). The dependency on proteasomal activity and on the cysteine-alanine motif demonstrates that the cODC1 degron induces proteolysis by the proteasome. In addition, we performed flow cytometry measurements of cells expressing tester constructs (see Additional file 1 Figure S1A, $B)$. These experiments confirmed our results obtained by immunoblotting (Figure 2A, C, E).

Next, we compared the destabilizing activity of the active cODC1 degron to that of the N-degron TDegF. We performed fluorescence microscopy to visualize the GFP and RFP fluorescence of the constructs GFPcODC1-TDegF-RFP and GFP-TDegF-RFP after pTEV $^{+}$ 


\section{A}

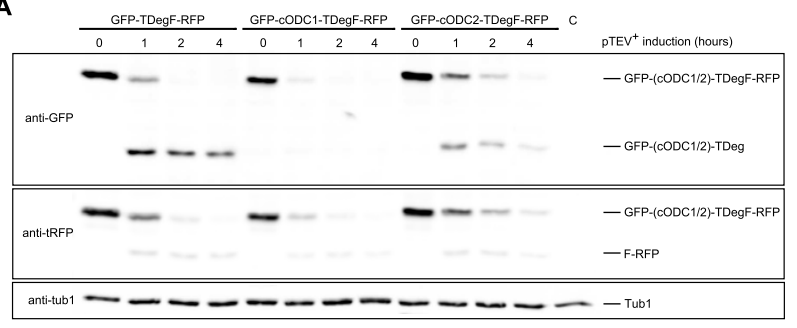

B

PTEV

PTEV
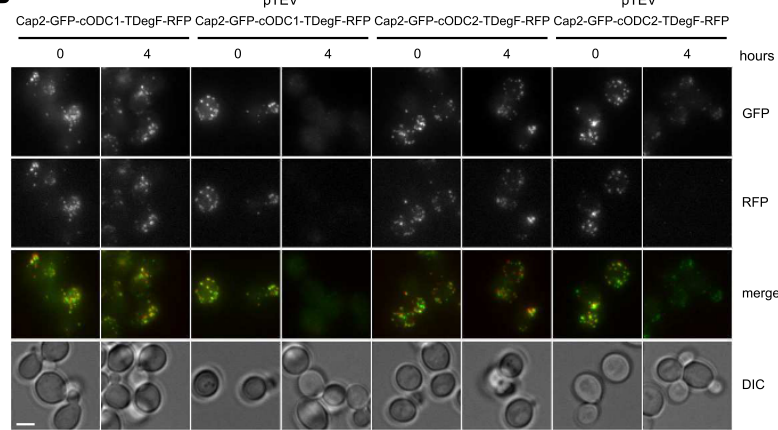

C

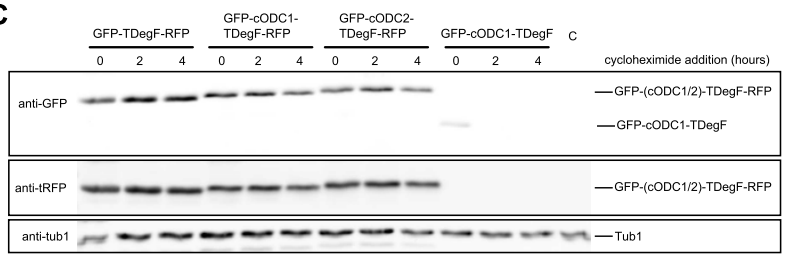

D

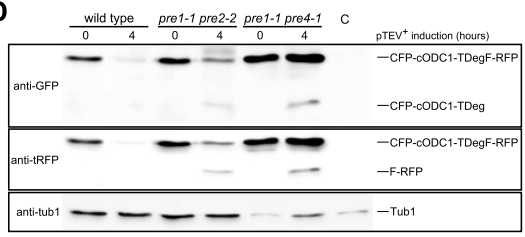

E

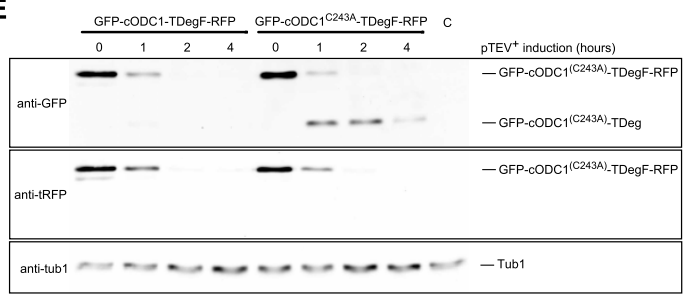

Figure 2 Control of protein abundance using the bidirectional degron. (A) The conditional C-degron included in CODC1-TDegF induces rapid protein depletion. The plasmid encoded constructs GFP-TDegF-RFP, GFP-CODC1-TDegF-RFP, and GFP-CODC2-TDegF-RFP were expressed in yeast cells (YCT1169) using the constitutive ADH1 promoter. Cells without a construct (lane C) served as control of antibody specificity. Expression of the PTEV $^{+}$protease was induced by addition of galactose (2\% final concentration). Samples of logarithmically growing yeast cells were taken at the indicated time points and subjected to western blotting. For detection, anti-GFP, anti-tRFP, and anti-tub1 (loading control) antibodies were used. Positions of cleaved and uncleaved species are indicated in the figure. (B) Observation of Cap2 depletion by live-cell imaging. CAP2-GFPCODC1-TDegF-RFP or CAP2-GFP-CODC2-TDegF-RFP (chromosomally encoded) was expressed in strains containing or lacking the gene encoding for the $\mathrm{pTEV}^{+}$protease. Images (maximum intensity projections shown) were recorded before and after 4 hours of pTEV ${ }^{+}$protease production. Bar size, $2.5 \mu \mathrm{m}$. (C) Translation shut-off experiment to measure the destabilizing properties of the dormant degrons in GFP-TDegF-RFP, GFP-CODC1TDegF-RFP, GFP-CODC2-TDegF-RFP, and GFP-CODC1-TDegF (plasmid encoded). The same conditions were used as described in A. Galactose and the translation elongation inhibitor cycloheximide were added at time point 0 hours. (D) Proteasomal activity is necessary for protein depletion mediated by the bidirectional degron. Plasmid encoded CFP-CODC1-TDegF-RFP was expressed in wild type and proteasomal mutant cells. Expression of the TTEV $^{+}$protease (plasmid encoded) was induced by the addition of galactose. Cells were kept at $30^{\circ} \mathrm{C}$ during the experiment (semi-permissive conditions for the proteasomal mutants). Samples were prepared as described in A. (E) Destabilizing activity of the conditional C-degron in CODC1-TDegF depends on the cysteine-alanine motif. The plasmid encoded constructs GFP-CODC1-TDegF-RFP and GFPCODC1 ${ }^{\text {C243A }}$-TDegF-RFP were expressed in yeast cells using the constitutive ADH1 promoter. Experimental procedure as described in A. 
protease cleavage. Only cells containing the cODC1 construct showed rapid loss of GFP fluorescence, whereas RFP fluorescence decreased in both cases (Figure 3A). Quantification of the images revealed that GFP-cODC1-TDeg was depleted as quickly as F-RFP (Figure 3B). Our experiments with diverse tester proteins demonstrate that the bidirectional degron cODC1TDegF induces rapid target protein depletion after TEV protease cleavage regardless if placed at the $\mathrm{N}$ - or $\mathrm{C}$-terminus or inside the protein.

As shown in Figure 1D, the TIPI system is not restricted to complete destabilization of proteins. Constructs containing cODC1-TDegM and TDegM could be used to degrade only a single domain and to separate two domains without destabilization, respectively. As expected, proteolytic cleavage of GFP-cODC1-TDegMRFP resulted in destabilized GFP-CODC1-TDeg and stable M-RFP whereas GFP-TDegM-RFP was split in two stable fluorescent proteins (Figure 3C).

In summary, our results demonstrate that it is possible to create quasi artificial, conditional degrons with distinct properties based on the features of the cODC degron. The newly developed cODC degrons induce rapid degradation and their destabilizing activities depend solely on proteolytic cleavage of the bidirectional degron by the $\mathrm{pTEV}^{+}$protease. The less destabilizing cODC2-TDegF tag might be useful to reduce target protein levels without complete depletion. Weak degrons have been used before to measure transcription [41] or proteasomal activity [42]. The data obtained with
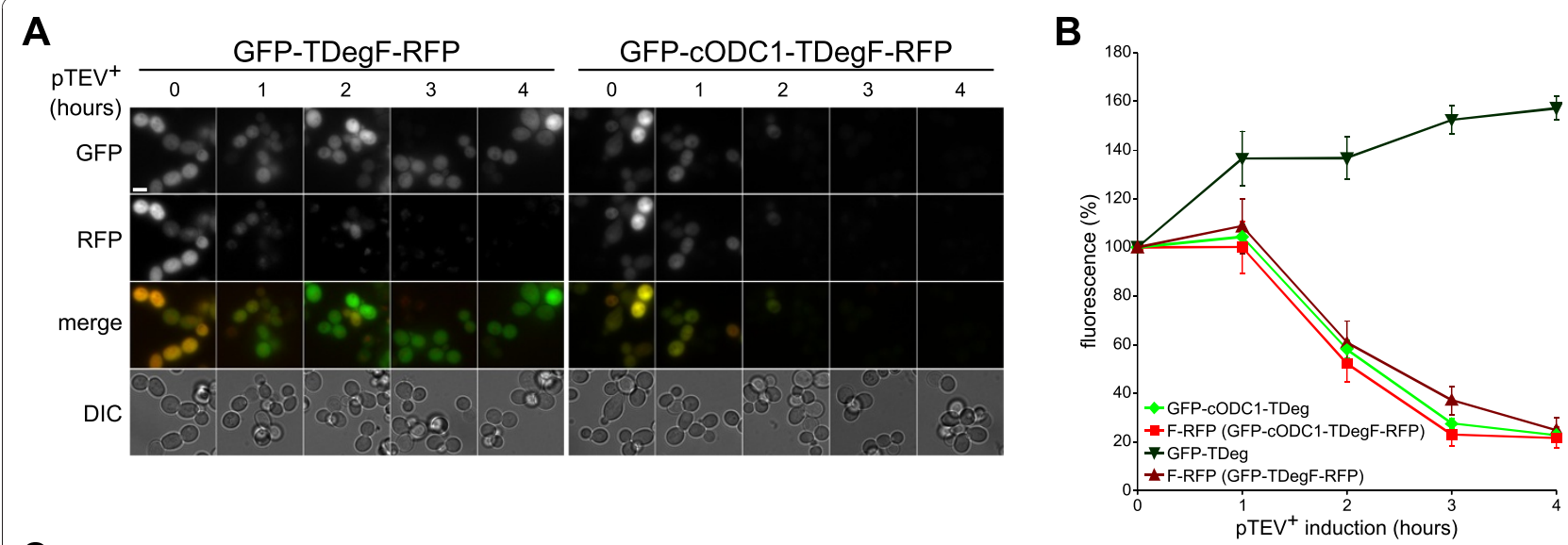

C

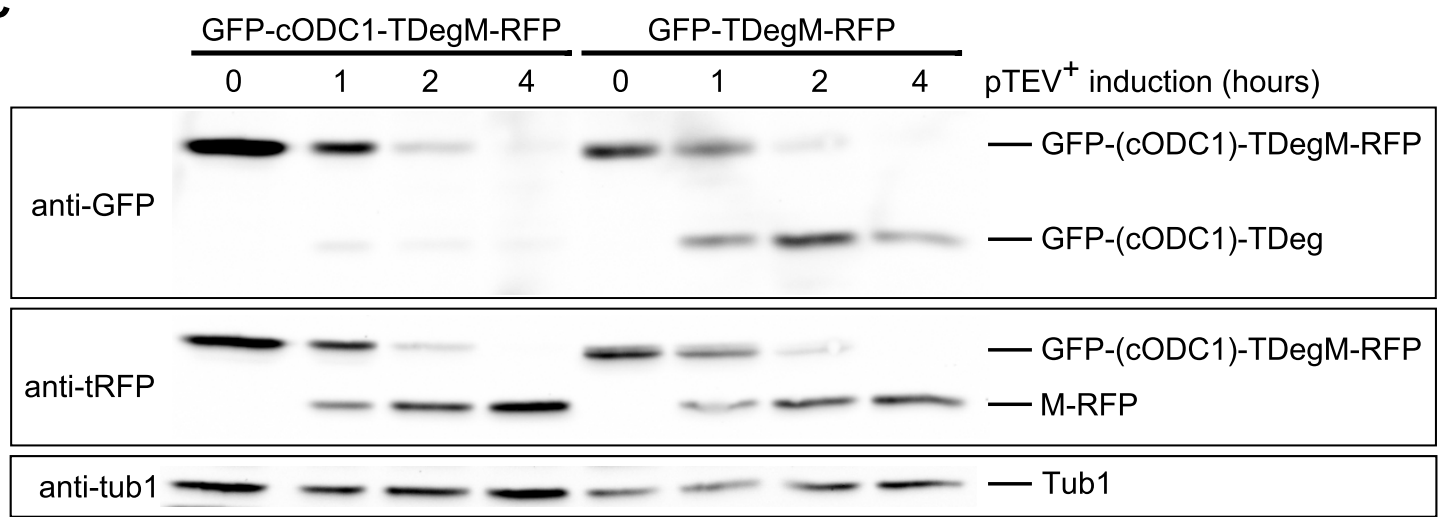

Figure 3 Control of protein stability using combinations of stabilizing and destabilizing sequences. (A) Live-cell imaging of cODC1mediated destabilization of GFP. Plasmid encoded GFP-TDegF-RFP and GFP-CODC1-TDegF-RFP were expressed constitutively under control of the ADH1 promoter in yeast cells (YCT1169). Images of the cells were taken at the indicated time points after induction of pTEV ${ }^{+}$protease expression. Bar size, $5 \mu \mathrm{m}$. (B) Kinetics of cODC1-mediated destabilization of GFP. Fluorescence intensities of the fragments, which were generated by TEV protease cleavage, were plotted over time. Images recorded for the experiment shown in A were used for automated quantitative image analysis to measure intracellular GFP and RFP fluorescence in 1000 to 3000 cells per strain (error bars indicate the standard error of the mean). The fragment GFP-TDeg is marked by inverted triangles, F-RFP (derived from GFP-TDegF-RFP) by triangles, GFP-cODC1-TDeg by diamonds, and F-RFP (derived from GFP-CODC1-TDegF-RFP) by squares. (C) Processing of tester proteins by the PTEV $^{+}$protease. The plasmid encoded constructs GFP-CODC1-TDegM-RFP and GFP-TDegM-RFP were expressed in yeast cells (YCT1169) using the constitutive ADH1 promoter. Samples of logarithmically growing yeast cells were taken at the indicated time points after induction of PTEV $^{+}$protease expression and subjected to western blotting. For detection, anti-GFP, anti-tRFP, and anti-tub1 (loading control) antibodies were used. Positions of cleaved and uncleaved species are indicated in the figure. 
combinations of stabilizing sequences, $\mathrm{N}$ - or C-degrons (Figure 2A and $3 \mathrm{C}$ ) show that the TIPI system provides a toolbox to control the fate of single domains in vivo.

\section{Creation of conditional mutants}

We assessed, whether activation of the bidirectional degron induces depletion of target proteins to levels, at which specific phenotypes become observable. Therefore, we inserted the GFP-cODC1-TDegF-RFP tag and the GFP-cODC1-TDegF tag chromosomally at the 3' end of the essential genes CDC14, CDC48, CYR1, KOG1, $C D C 20, M C M 1$, and $C D C 5$. The amino acid sequence of both tags is given in Figure S3 (see Additional file 1). We obtained conditional mutants for 5 of the 7 genes. No reduction of viability at restrictive conditions was observed for modified Kog1 and Cdc20, whereas GFPcODC1-TDegF-RFP and GFP-cODC1-TDegF-tagged Cdc48 and Cyr1 as well as Mcm1-GFP-cODC1-TDegF, Cdc5-GFP-cODC1-TDegF, and Cdc14-GFP-cODC1TDegF-RFP led to severe growth defects (Figure 4A, B). This demonstrates that the GFP-cODC1-TDegF-RFP and GFP-cODC1-TDegF tags can be used to create conditional mutants. A possible explanation for the differences between GFP-cODC1-TDegF-RFP and GFPcODC1-TDegF mutants might lie in changes of target protein levels induced by the GFP-cODC1-TDegF tag at permissive conditions, as we found that the GFPcODC1-TDegF construct is destabilized without TEV protease cleavage (Figure 2C). Indeed, steady state levels of the essential proteins modified with the GFP-cODC1TDegF tag were reduced in comparison with the corresponding GFP-tagged proteins (data not shown). However, growth of the mutants was only slightly affected at permissive conditions on glucose containing media (Figure 4B). A reason could be that most yeast proteins are synthesized at much higher levels than necessary for survival under lab conditions [43].

Additionally, we followed the cleavage of the fusion proteins by the $\mathrm{pTEV}^{+}$protease using immunoblotting. We observed cleavage in case of Cdc5, Cdc14, Cdc48, Cyr1, and Mcm1 (see Additional file 1 Figure S2). Due to the absence of clear signals for Cdc20-, or Kog1GFP-cODC1-TDegF (data not shown), it is not clear why creation of conditional mutants failed for these proteins.

We performed live-cell imaging with cells expressing Cdc14-GFP-cODC1-TDegF-RFP and Cdc48-GFPcODC1-TDegF-RFP fusion proteins. The Cdc14-GFPcODC1-TDegF-RFP fusion protein localized to the nucleolus, as expected, whereas Cdc48-GFP-cODC1TDegF-RFP mislocalized to several very bright spots (Figure 4C). The GFP and RFP fluorescence dropped down to roughly $10 \%$ of the initial levels in both cases within three hours after $p T E V^{+}$protease expression. The fluorescence did not change considerably in the absence of the protease (Figure 4C, D).

We checked the consequences of Cdc5, Cdc14, Cdc48, Cyr1, and Mcm1 depletion in vivo. All these proteins have essential functions during the yeast cell cycle, which were studied intensively with conventional mutants. Loss of activity results in each case in a specific defect. It was found that $c y r 1-1$ and $c d c 48$ - $t d$ mutants arrest with no bud at restrictive conditions $[44,45]$. A metaphase arrest was observed in the mcm1-110L mutant [46] as well as the TDegF-cdc14 mutant [25] and an anaphase arrest in case of $c d c 5$ temperature-sensitive mutants [47]. To compare the phenotypes obtained by protein depletion with conventional mutants, we checked for cell cycle phenotypes based on spindle morphology and bud-size in GFP-CODC1-TDegF mutants of CDC5, CDC14, CDC48, CYR1 and MCM1. We found that all GFP-cODC1-TDegF mutants accumulated in a specific cell cycle stage if the $\mathrm{pTEV}^{+}$protease was present (Figure 4E), matching the results, which were reported for other conditional mutants in the literature. In the absence of the $\mathrm{pTEV}^{+}$protease, we found no apparent difference to control cells (data not shown). Our data demonstrates that both, the GFP-cODC1TDegF-RFP and the GFP-cODC1-TDegF tag can be used to create conditional mutants. In addition, the GFP-cODC1-TDegF-RFP tag is useful for applications, which ask for observation of substrate cleavage using live-cell imaging.

\section{Creation of developmental process-specific mutants}

Another interesting application for the TIPI system would be to use it as a method to deplete target proteins at a specific stage during a developmental process. We chose the developmental program of sporulation in S. cerevisiae as model process. Sporulation is initiated by starvation in yeast and consists of the meiotic cell divisions followed by spore formation [48]. To restrict the expression of the $p T E V^{+}$protease to meiosis, we used the $I M E 2$ promoter, which is active only during sporulation [49]. As it is known that loss of Cdc14 or Cdc5 results in defects during the first meiotic division $[50,51]$, we selected them as target proteins for $\mathrm{N}$-degron and $\mathrm{C}$-degron dependent depletion, respectively. We fused the TDegF tag under control of the $C Y C 1$ promoter, which is repressed during meiosis and spore formation [52], to the 5'-end of CDC14. This led to reduction of GFP-TDegF-Cdc14 levels, whereas additional expression of the $p T E V^{+}$protease led to complete absence (Figure 5A) and a defect in spore formation, most likely due to a block in the first meiotic division (Figure 5B). We added the GFP-cODC1-TDegF tag to the 3'-end of $C D C 5$ and subjected the cells to sporulation conditions. Surprisingly, the Cdc5-GFP-cODC1- 


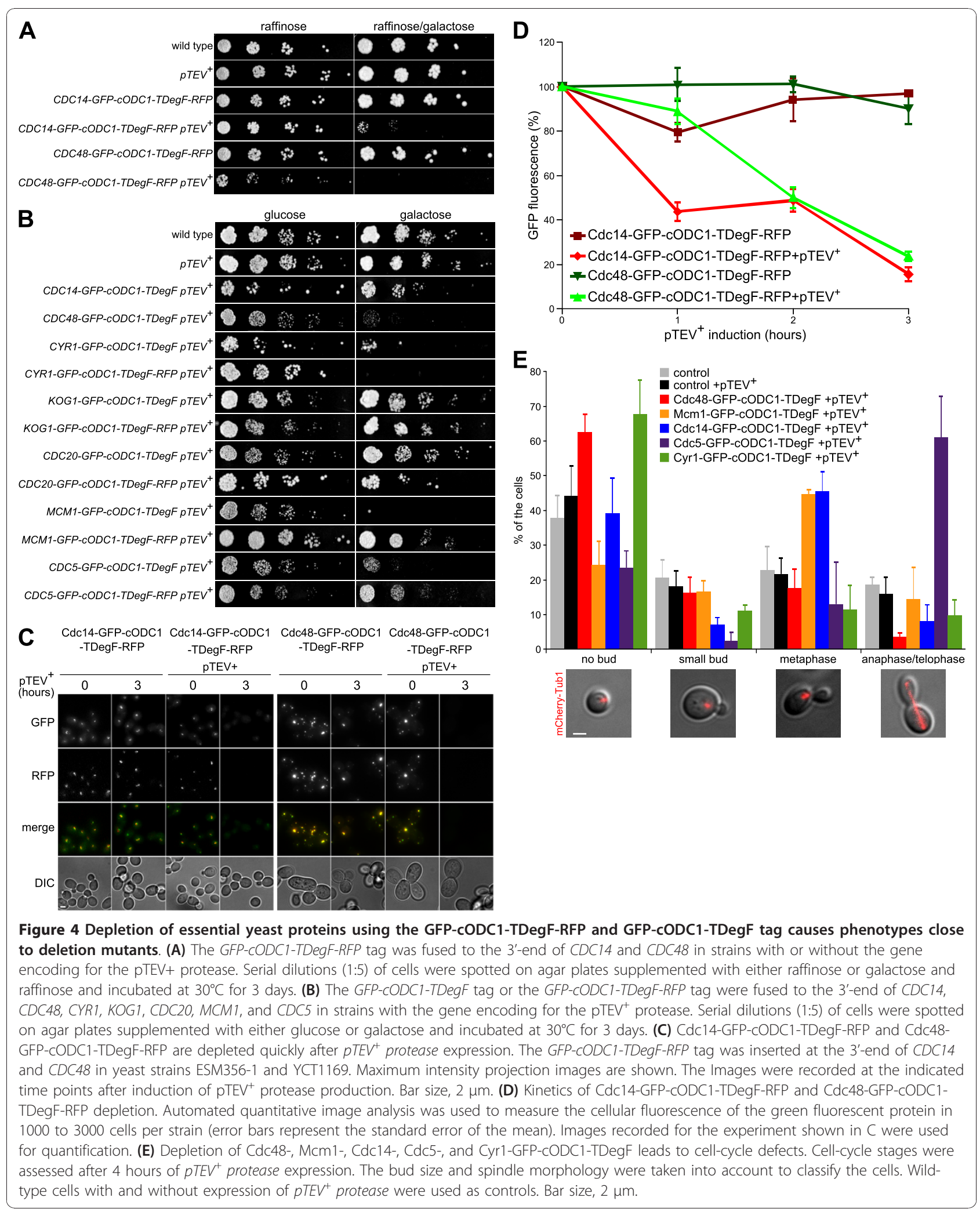



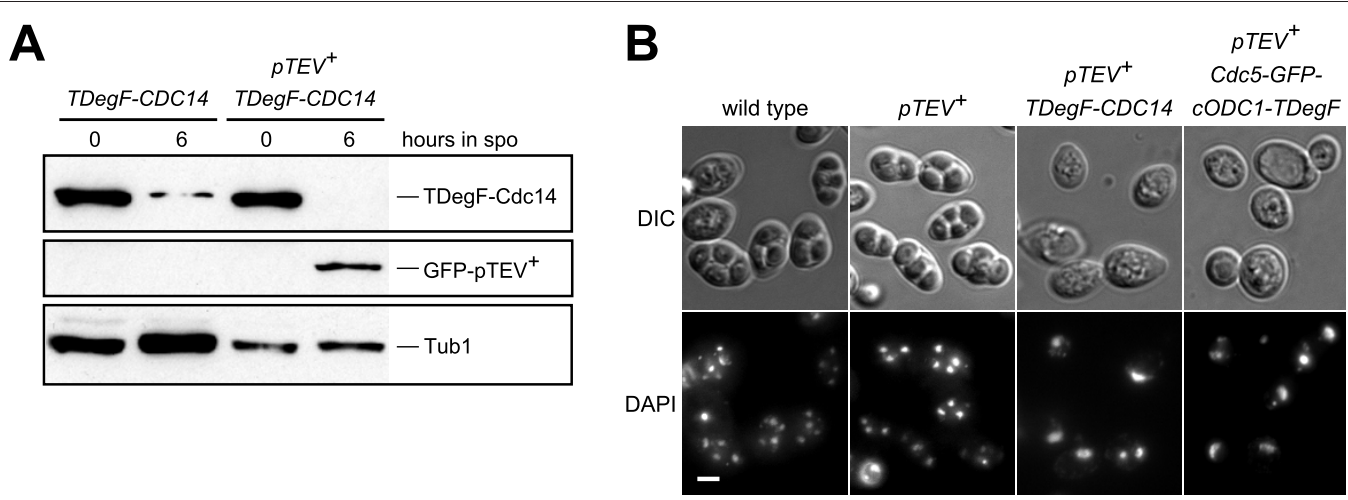

Figure 5 Developmental process-specific depletion of essential proteins. (A) Meiosis-specific depletion of Cdc14. Yeast cells with $P_{C Y C 1}-G F P-$ TDegF-CDC14 were subjected to sporulation in the absence or presence of $P_{I M E 2}-G F P-p T E V^{+}$. Samples were taken at the indicated time points after induction of meiosis due to shift on sporulation medium (spo). The proteins (GFP-TDegF-Cdc14 and GFP-pTEV+ protease) were detected with antibodies directed against GFP; tubulin was used as loading control. (B) Depletion of Cdc14 or Cdc5 leads to a block during meiosis I. Yeast cells sporulated for 24 hours were stained with Hoechst 33342 to visualize the DNA. Bright field (DIC) and fluorescent (DAPI) images (maximum intensity projections) are shown. The genotypes of the strains are indicated in the figure. The CDC5-GFP-CODC1-TDegF cells contained a high copy plasmid with PIME2 $_{\text {GFP-PTEV }}{ }^{+}$. Bar size, $2 \mu \mathrm{m}$.

TDegF containing cells sporulated in the presence of the $\mathrm{pTEV}^{+}$protease (data not shown). However, high-level production of the $\mathrm{pTEV}^{+}$protease achieved by transformation of the cells with a high-copy plasmid containing the $p T E V^{+}$protease gene resulted in a block of sporulation in the meiotic prophase (Figure 5B). High-level production of the $\mathrm{pTEV}^{+}$protease alone did not change sporulation behavior of wild type yeast cells (data not shown). Thus, the TIPI system is a valuable technique to study developmental processes. Timely expression of the TEV protease during a specific cell cycle stage or a developmental process allows control of target protein abundance during that stage.

\section{Conclusions}

The bidirectional degron can be fused to a target protein N-terminally, C-terminally or placed internally. Activation of the degrons is achieved by cleavage with the tobacco etch virus protease, resulting in quick degradation of the target protein. Thus the method provides a high degree of freedom to the user in terms of target protein modification with the bidirectional degron and control of TEV protease production. However, the usage might be constrained for some applications. The GFP-cODC1-TDegF-RFP tag is rather big as it contains two different fluorescent proteins. On the one hand this allows following cleavage of the tag by live-cell imaging, but on the other hand it limits the repertoire of additional fluorophores. The smaller GFP-cODC1-TDegF tag, which lacks the RFP, destabilizes the target protein in the absence of the TEV protease resulting in a reduction of protein levels. Even some of the GFP-cODC1TDegF-RFP-tagged proteins exhibited reduced steady state levels (data not shown), but less pronounced than in case of the GFP-cODC1-TDegF-modified proteins. As we did not observe destabilization of the GFP-cODC1TDegF-RFP tester protein without TEV protease cleavage (Figure $2 \mathrm{C}$ ), this decrease might be caused by the leakiness of the GAL1 promoter [53] resulting in weak expression of the TEV protease. Another possible explanation might be that the GFP-cODC1-TDegF-RFP tag changes the efficiency of protein synthesis. The normal or only slightly affected growth of the conditional mutants at permissive conditions (Figure 4A, B) could be explained by the observation that most yeast proteins are synthesized in excess [43]. A new construct, lacking the GFP and keeping the RFP, might be a way to decrease the size of the construct and to circumvent the problem of reduced protein abundance.

The C- and N-degron we used to construct the bidirectional degron are based on conserved degradation mechanisms and are known to work in higher eukaryotes $[17,54]$. Therefore, protein depletion utilizing the TIPI system should be possible in animals as well, as it has been shown already that cell type specific usage of the TEV protease in Drosophila melanogaster is feasible [55]. In synthetic biology approaches the TIPI system might be employed to implement protein destabilization into regulatory circuits or to cleave a protein artificially. Advantageous is that the system is reversible [25] and allows genetic control of protein stability. Furthermore, the degradation tag and the TEV protease are heterologous proteins or protein domains. Therefore, potentially harmful interactions with cellular components are minimized. Previously, chromosome separation has been studied using TEV protease cleavage [55,56], but without incorporation of conditional degrons. Our work enhances the existing techniques, as it provides the 
possibility to inflict different fates to the $\mathrm{N}$ - and C-terminus of the target protein after cleavage. Destabilization of one or the other is achieved using the right combination of degron and stabilizing sequence (Figure 1D). This might be a way to probe for regulatory domains or to create separation-of-function-mutants at protein level. These applications are unique to the TIPI system and exemplify its value as a cell biology tool to manipulate protein abundance and activity in vivo.

\section{Methods}

\section{Yeast strains, growth conditions and plasmid constructions}

The yeast strains were derived from the S288C strain ESM356-1 [57], WCG4a [40] or the SK-1 strain YKS32 [58] as indicated in the Table S1 (see Additional file 2). Standard methods were used to construct yeast strains; standard preparations of media were used for growth [59]. Gene tagging with PCR products was performed as described [53]. Yeast cells were grown at $30^{\circ} \mathrm{C}$, cells used for the experiment shown in Figure 2D (WCG4a, WCG4a-11/22, and YHI29/ 14) were grown at $25^{\circ} \mathrm{C}$ and shifted to $30^{\circ} \mathrm{C}$ for the time of the experiment. Growth tests were performed on rich media supplemented with glucose, raffinose or galactose ( $2 \%$ final concentration), as indicated for the experiments. Cells were grown in low-fluorescence media [60] for fluorescent microscopy experiments. Plasmids were constructed by standard procedures [61], details and sequences of the used vectors are available on request. The plasmids are listed in Table S2 (see Additional file 2).

\section{Immunoblotting and cycloheximide chase experiment}

The immunoblotting experiments were performed essentially as described [25]. The amount of yeast culture corresponding to $1 \mathrm{OD}_{600}$ of cells was collected at each time point. Cells were grown in synthetic complete media supplemented with raffinose (2\%), galactose was added after collection of the first sample (time 0 hours) to induce the $\mathrm{pTEV}^{+}$protease production. Samples were subjected to alkaline lysis and TCA precipitation, SDS-PAGE and blotting. Commercially available antibodies against GFP (Santa Cruz biotechnology, Santa Cruz, USA), tRFP (Biocat, Heidelberg, Germany) and HRPO-coupled antibodies directed against mouse or rabbit (Santa Cruz biotechnology, Santa Cruz, USA) were used to detect tester proteins. The rabbit anti-tubulin antibody (a kind gift of $\mathrm{M}$. Knop, EMBL Heidelberg) was used to detect tubulin, which served as a loading control. Chemiluminescence was detected using a western blot imager (INTAS Science Imaging Instruments, Göttingen, Germany). Signal intensities were quantified with the software ImageJ [62]. To perform the translation inhibition experiment (Figure 2C), cycloheximide was added to the cultures at a final concentration of $0.1 \mathrm{mg} / \mathrm{ml}$. Samples were collected at the times indicated in the figure and subjected to alkaline lysis and TCA precipitation.

\section{Microscopy}

Live-cell imaging was performed as described [25,60] using a Zeiss Axiovert 200 equipped with a Hamamatsu camera, DAPI, EGFP and rhodamine filter sets and a 63 times Plan Apochromat oil lens (NA 1.4). Images were collected as z-stacks with $0.5 \mu \mathrm{m}$ spacing (Figure $2 \mathrm{~B}, 4 \mathrm{C}$ ) or single images (Figure $3 \mathrm{~A}$ ). Quantitative imaging was performed as follows: Logarithmically grown yeast cells were adhered to glass-bottom-dishes (MatTek Corporation, Ashland, USA) treated with concanavalin A. Synthetic complete media with raffinose was added and $\mathrm{z}$-stack images with $0.5 \mu \mathrm{m}$ spacing or single images were taken at the time points indicated in the figures. Galactose ( $2 \%$ final concentration) was added to the medium after collection of the 0 hours image series. Images of the cells were collected in the bright field and fluorescent channels indicated in the figures. Quantification of the images was done with the software Image (version $1.40 \mathrm{~g}$ ). Slightly outof-focus bright field images were used to detect the cell outlines. The commands "find edges", "gaussian blur (radius = 2)", "contrast setting" and "make binary" were used on these images to generate binary images of cell outlines. With the commands "fill holes" and "create selection" a mask covering whole cells was obtained. These masks were used to measure mean signal intensities in the images of the fluorescence channels. Maximum projections of z-stacks were used for the measurements in case $z$-stack images were recorded.

\section{Growth assays}

Serial dilutions (1:5) of cells grown in YP + raffinose or YPD were placed on YP + raffinose, YP + raffinose and galactose, YPD or YP + galactose plates (as indicated in Figure 4). Cells corresponding to $0.05 \mathrm{OD}_{600}$ were diluted in $1 \mathrm{ml}$ of water. Subsequently, 4 dilutions were made and $3 \mu \mathrm{l}$ of each dilution were spotted on the plates. Pictures of the spots were taken after incubation for 3 days at $30^{\circ} \mathrm{C}$.

\section{FACS measurements}

The cells were grown to mid-log phase in low fluorescence media containing raffinose. The culture was divided in two; one was treated with sodium azide (10 $\mathrm{mM}$ final concentration) and kept on ice until the measurement in the flow cytometer (sample -TEV). The other half of the culture was supplemented with galactose. The cells were incubated with shaking for 4 hours at $30^{\circ} \mathrm{C}$ and sodium azide was added (sample + TEV). The cells were analyzed in a FACSCalibur flow 
cytometer (Becton Dickinson) equipped with a $488 \mathrm{~nm}$ argon laser and a 530/30 band-pass filter for GFP fluorescence detection. Around 85000 events were collected for each sample; all events were used to create the graphs and to calculate the median fluorescence.

\section{Sporulation}

Synchronous sporulation was performed using the presporulation treatment as described [63]. Potassium acetate $(1 \%)$ was used as sporulation medium. Cells were stained with Hoechst 33342 after fixation with 70\% ethanol.

\section{Additional material}

Additional file 1: FACS measurements of tester constructs, depletion of essential yeast proteins and sequence of the GFPCODC1-TDegF and GFP-CODC1-TDegF-RFP tags. FACS measurements. We performed flow cytometry measurements with cells expressing GFP-

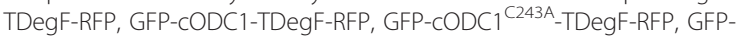
CODC2-TDegF-RFP, and GFP-CODC1-TDegF to measure the GFP fluorescence in the absence and the presence of the $\mathrm{pTEV}^{+}$protease. We found background fluorescence in the absence of the protease in case of the GFP-CODC1-TDegF construct. This argues for a destabilization of the construct independently of the CODC degron. Nevertheless, we detected robust GFP fluorescence in the other constructs in the absence of the protease. Cells producing the $\mathrm{pTEV}^{+}$protease showed nearly background fluorescence in the CODC containing constructs and reduced GFP fluorescence in case of the constructs GFP-TDegF-RFP and

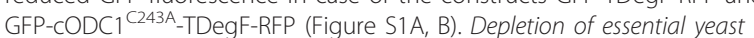
proteins. We checked the production and proteolytic cleavage of the fusion proteins Cdc14- and Cdc48-GFP-CODC1-TDegF-RFP as well as the Cdc5-, Cyr1-, and Mcm1-GFP-cODC1-TDegF by immunoblotting. It was possible to detect all fusion proteins in crude yeast extracts in the absence of the $\mathrm{pTEV}^{+}$protease. Presence of the protease resulted in partial or complete depletion of the target proteins (Figure S2). Sequence of the GFP-CODC1-TDegF and GFP-CODC1-TDegF-RFP tags. The amino acid sequence of the GFP-CODC1-TDegF and the GFP-CODC1-TDegF-RFP tags is given (Figure S3). The sequences of yeast enhanced GFP and mKate have been published $[64,65]$.

Additional file 2: Information on yeast strains (Table S1) and plasmids (Table S2) used for this study.

\section{Acknowledgements}

We thank M Knop and E Schiebel for their generous share of plasmids and reagents as well as $\mathrm{P}$ Yu for help with the flow cytometer. D Störmer is acknowledged for her excellent technical assistance and HU Mösch for helpful comments on the manuscript. MJ was supported by the DFG grant GK1216 "Intra and Intercellular Transport and Communication".

\section{Authors' contributions}

All authors were involved in generation of plasmids and yeast strains. MJ and $C R$ performed the sporulation experiments and helped to write the manuscript. CT conceived the study, carried out experiments and wrote the manuscript. All authors read and approved the final manuscript.

Received: 16 April 2010 Accepted: 29 December 2010

Published: 29 December 2010

\section{References}

1. Banaszynski LA, Wandless TJ: Conditional control of protein function. Chem Biol 2006, 13:11-21.
2. Breslow DK, Cameron DM, Collins SR, Schuldiner M, Stewart-Ornstein J, Newman HW, Braun S, Madhani HD, Krogan NJ, Weissman JS: A comprehensive strategy enabling high-resolution functional analysis of the yeast genome. Nat Methods 2008, 5:711-718.

3. Bulina ME, Chudakov DM, Britanova OV, Yanushevich YG, Staroverov DB, Chepurnykh TV, Merzlyak EM, Shkrob MA, Lukyanov S, Lukyanov KA: A genetically encoded photosensitizer. Nat Biotechnol 2006, 24:95-99.

4. Elbashir SM, Harborth J, Lendeckel W, Yalcin A, Weber K, Tuschl T: Duplexes of 21-nucleotide RNAs mediate RNA interference in cultured mammalian cells. Nature 2001, 411:494-498.

5. Haruki H, Nishikawa J, Laemmli UK: The anchor-away technique: rapid, conditional establishment of yeast mutant phenotypes. Mol Cell 2008, 31:925-932.

6. Mnaimneh S, Davierwala AP, Haynes J, Moffat J, Peng WT, Zhang W, Yang X, Pootoolal J, Chua G, Lopez A, Trochesset M, Morse D, Krogan NJ, Hiley SL, Li Z, Morris Q, Grigull J, Mitsakakis N, Roberts CJ, Greenblatt JF, Boone C, Kaiser CA, Andrews BJ, Hughes TR: Exploration of essential gene functions via titratable promoter alleles. Cell 2004, 118:31-44.

7. Sauer B: Cre/lox: one more step in the taming of the genome. Endocrine 2002, 19:221-228.

8. Schrader EK, Harstad KG, Matouschek A: Targeting proteins for degradation. Nat Chem Biol 2009, 5:815-822.

9. Nishimura K, Fukagawa T, Takisawa H, Kakimoto T, Kanemaki M: An auxinbased degron system for the rapid depletion of proteins in nonplant cells. Nat Methods 2009, 6:917-922.

10. Sakamoto KM, Kim KB, Kumagai A, Mercurio F, Crews CM, Deshaies RJ: Protacs: chimeric molecules that target proteins to the Skp1-Cullin-F box complex for ubiquitination and degradation. Proc Natl Acad Sci USA 2001, 98:8554-8559.

11. Banaszynski LA, Chen LC, Maynard-Smith LA, Ooi AG, Wandless TJ: A rapid, reversible, and tunable method to regulate protein function in living cells using synthetic small molecules. Cell 2006, 126:995-1004.

12. Levy F, Johnston JA, Varshavsky A: Analysis of a conditional degradation signal in yeast and mammalian cells. Eur J Biochem 1999, 259:244-252.

13. Grilly C, Stricker J, Pang WL, Bennett MR, Hasty J: A synthetic gene network for tuning protein degradation in Saccharomyces cerevisiae. Mol Syst Biol 2007, 3:127.

14. Bachmair A, Finley D, Varshavsky A: In vivo half-life of a protein is a function of its amino-terminal residue. Science 1986, 234:179-186.

15. Varshavsky A: The N-end rule. Cell 1992, 69:725-735.

16. Varshavsky A: Ubiquitin fusion technique and related methods. Methods Enzymol 2005, 399:777-799.

17. Mogk A, Schmidt R, Bukau B: The N-end rule pathway for regulated proteolysis: prokaryotic and eukaryotic strategies. Trends Cell Biol 2007, 17:165-172.

18. Varshavsky A: The N-end rule: functions, mysteries, uses. Proc Natl Acad Sci USA 1996, 93:12142-12149.

19. Dohmen RJ, Wu P, Varshavsky A: Heat-inducible degron: a method for constructing temperature-sensitive mutants. Science 1994, 263:1273-1276.

20. Araki Y, Lau CK, Maekawa H, Jaspersen SL, Giddings TH Jr, Schiebel E, Winey M: The Saccharomyces cerevisiae spindle pole body (SPB) component Nbp1p is required for SPB membrane insertion and interacts with the integral membrane proteins Ndc1p and Mps2p. Mol Biol Cell 2006, 17:1959-1970.

21. Kadota J, Yamamoto T, Yoshiuchi S, Bi E, Tanaka K: Septin ring assembly requires concerted action of polarisome components, a PAK kinase Cla4p, and the actin cytoskeleton in Saccharomyces cerevisiae. Mol Biol cell 2004, 15:5329-5345.

22. Sanchez-Diaz A, Kanemaki M, Marchesi V, Labib K: Rapid depletion of budding yeast proteins by fusion to a heat-inducible degron. SCi STKE 2004, 2004:PL8.

23. Wang X, Ira G, Tercero JA, Holmes AM, Diffley JF, Haber JE: Role of DNA replication proteins in double-strand break-induced recombination in Saccharomyces cerevisiae. Mol Cell Biol 2004, 24:6891-6899.

24. Wu WH, Wu CH, Ladurner A, Mizuguchi G, Wei D, Xiao H, Luk E, Ranjan A, Wu C: $\mathrm{N}$ terminus of Swr 1 binds to histone $\mathrm{H} 2 \mathrm{AZ}$ and provides a platform for subunit assembly in the chromatin remodeling complex. $J$ Biol Chem 2009, 284:6200-6207.

25. Taxis C, Stier G, Spadaccini R, Knop M: Efficient protein depletion by genetically controlled deprotection of a dormant N-degron. Mol Syst Biol 2009, 5:267. 
26. Takeuchi J, Chen H, Hoyt MA, Coffino P: Structural elements of the ubiquitin-independent proteasome degron of ornithine decarboxylase. Biochem J 2008, 410:401-407.

27. Murakami Y, Matsufuji S, Kameji T, Hayashi S, Igarashi K, Tamura T, Tanaka K, Ichihara A: Ornithine decarboxylase is degraded by the $26 \mathrm{~S}$ proteasome without ubiquitination. Nature 1992, 360:597-599.

28. Hoyt MA, Zhang M, Coffino P: Ubiquitin-independent mechanisms of mouse ornithine decarboxylase degradation are conserved between mammalian and fungal cells. J Biol Chem 2003, 278:12135-12143.

29. Takeuchi J, Chen H, Coffino P: Proteasome substrate degradation requires association plus extended peptide. EMBO J 2007, 26:123-131.

30. Li X, Coffino P: Degradation of ornithine decarboxylase: exposure of the C-terminal target by a polyamine-inducible inhibitory protein. Mol Cell Biol 1993, 13:2377-2383.

31. DeScenzo RA, Minocha SC: Modulation of cellular polyamines in tobacco by transfer and expression of mouse ornithine decarboxylase cDNA. Plant Mol Biol 1993, 22:113-127.

32. Matsuzawa S, Cuddy M, Fukushima T, Reed JC: Method for targeting protein destruction by using a ubiquitin-independent, proteasomemediated degradation pathway. Proc Natl Acad Sci USA 2005, 102:14982-14987.

33. Ghoda L, van Daalen Wetters T, Macrae M, Ascherman D, Coffino P: Prevention of rapid intracellular degradation of ODC by a carboxylterminal truncation. Science 1989, 243:1493-1495.

34. Loetscher $P$, Pratt $G$, Rechsteiner $M$ : The $C$ terminus of mouse ornithine decarboxylase confers rapid degradation on dihydrofolate reductase. Support for the pest hypothesis. J Biol Chem 1991, 266:11213-11220.

35. Huh WK, Falvo JV, Gerke LC, Carroll AS, Howson RW, Weissman JS, O'Shea EK: Global analysis of protein localization in budding yeast. Nature 2003, 425:686-691

36. Spadaccini R, Reidt U, Dybkov O, Will C, Frank R, Stier G, Corsini L, Wahl MC, Luhrmann R, Sattler M: Biochemical and NMR analyses of an SF3b155p14-U2AF-RNA interaction network involved in branch point definition during pre-mRNA splicing. Rna 2006, 12:410-425.

37. Prakash S, Tian L, Ratliff KS, Lehotzky RE, Matouschek A: An unstructured initiation site is required for efficient proteasome-mediated degradation. Nat Struct Mol Biol 2004, 11:830-837.

38. Tompa P, Prilusky J, Silman I, Sussman JL: Structural disorder serves as a weak signal for intracellular protein degradation. Proteins 2008, 71:903-909.

39. Gerlinger UM, Guckel R, Hoffmann M, Wolf DH, Hilt W: Yeast cycloheximide-resistant $\mathrm{crl}$ mutants are proteasome mutants defective in protein degradation. Mol Biol Cell 1997, 8:2487-2499.

40. Heinemeyer W, Gruhler A, Mohrle V, Mahe Y, Wolf DH: PRE2, highly homologous to the human major histocompatibility complex-linked RING10 gene, codes for a yeast proteasome subunit necessary for chrymotryptic activity and degradation of ubiquitinated proteins. J Biol Chem 1993, 268:5115-5120.

41. Hackett EA, Esch RK, Maleri S, Errede B: A family of destabilized cyan fluorescent proteins as transcriptional reporters in S. cerevisiae. Yeast 2006, 23:333-349.

42. Dantuma NP, Lindsten K, Glas R, Jellne M, Masucci MG: Short-lived green fluorescent proteins for quantifying ubiquitin/proteasome-dependent proteolysis in living cells. Nat Biotechnol 2000, 18:538-543.

43. Springer M, Weissman JS, Kirschner MW: A general lack of compensation for gene dosage in yeast. Mol Syst Biol 2010, 6:368.

44. Fu X, Ng C, Feng D, Liang C: Cdc48p is required for the cell cycle commitment point at Start via degradation of the G1-CDK inhibitor Far1p. J Cell Biol 2003, 163:21-26.

45. Matsumoto K, Uno I, Ishikawa T: Control of cell division in Saccharomyces cerevisiae mutants defective in adenylate cyclase and cAMP-dependent protein kinase. Exp Cell Res 1983, 146:151-161

46. Elble R, Tye BK: Chromosome loss, hyperrecombination, and cell cycle arrest in a yeast mcm1 mutant. Mol Biol Cell 1992, 3:971-980.

47. Hartwell LH, Mortimer RK, Culotti J, Culotti M: Genetic Control of the Cell Division Cycle in Yeast: V. Genetic Analysis of cdc Mutants. Genetics 1973, 74:267-286.

48. Kassir Y, Adir N, Boger-Nadjar E, Raviv NG, Rubin-Bejerano I, Sagee S, Shenhar G: Transcriptional regulation of meiosis in budding yeast. Int Rev Cytol 2003, 224:111-171.
49. Inai T, Yukawa M, Tsuchiya E: Interplay between chromatin and transacting factors on the IME2 promoter upon induction of the gene at the onset of meiosis. Mol Cell Biol 2007, 27:1254-1263.

50. Clyne RK, Katis VL, Jessop L, Benjamin KR, Herskowitz I, Lichten M, Nasmyth K: Polo-like kinase Cdc5 promotes chiasmata formation and cosegregation of sister centromeres at meiosis I. Nat Cell Biol 2003, 5:480-485

51. Marston $\mathrm{AL}$, Lee $\mathrm{BH}, \mathrm{Amon} \mathrm{A}$ : The $\mathrm{Cdc} 14$ phosphatase and the FEAR network control meiotic spindle disassembly and chromosome segregation. Dev Cell 2003, 4:711-726.

52. Chu S, DeRisi J, Eisen M, Mulholland J, Botstein D, Brown PO, Herskowitz I: The transcriptional program of sporulation in budding yeast. Science 1998, 282:699-705.

53. Janke C, Magiera MM, Rathfelder N, Taxis C, Reber S, Maekawa H, MorenoBorchart A, Doenges G, Schwob E, Schiebel E, Knop M: A versatile toolbox for PCR-based tagging of yeast genes: new fluorescent proteins, more markers and promoter substitution cassettes. Yeast 2004, 21:947-962.

54. Hoyt MA, Coffino P: Ubiquitin-free routes into the proteasome. Cell Mol Life Sci 2004, 61:1596-1600.

55. Pauli A, Althoff F, Oliveira RA, Heidmann S, Schuldiner O, Lehner CF, Dickson BJ, Nasmyth K: Cell-type-specific TEV protease cleavage reveals cohesin functions in Drosophila neurons. Dev Cell 2008, 14:239-251.

56. Uhlmann F, Wernic D, Poupart MA, Koonin EV, Nasmyth K: Cleavage of cohesin by the $C D$ clan protease separin triggers anaphase in yeast. Cell 2000, 103:375-386.

57. Pereira G, Tanaka TU, Nasmyth K, Schiebel E: Modes of spindle pole body inheritance and segregation of the Bfa1p-Bub2p checkpoint protein complex. EMBO J 2001, 20:6359-6370.

58. Knop M, Strasser K: Role of the spindle pole body of yeast in mediating assembly of the prospore membrane during meiosis. EMBO J 2000, 19:3657-3667.

59. Sherman F: Getting started with yeast. Methods Enzymol 2002, 350:3-41

60. Taxis C, Maeder C, Reber S, Rathfelder N, Miura K, Greger K, Stelzer EH, Knop M: Dynamic organization of the actin cytoskeleton during meiosis and spore formation in budding yeast. Traffic 2006, 7:1628-1642.

61. Ausubel FM, Kingston RE, Seidman FG, Struhl K, Moore DD, Brent R, Smith FA, Ed: Current Protocols in Molecular Biology. New York, USA: John Wiley and Sons; 1995.

62. Collins TJ: ImageJ for microscopy. Biotechniques 2007, 43:25-30.

63. Taxis C, Keller P, Kavagiou Z, Jensen LJ, Colombelli J, Bork P, Stelzer EH, Knop M: Spore number control and breeding in Saccharomyces cerevisiae: a key role for a self-organizing system. J Cell Biol 2005, 171:627-640.

64. Cormack BP, Bertram G, Egerton M, Gow NA, Falkow S, Brown AJ: Yeastenhanced green fluorescent protein (yEGFP)a reporter of gene expression in Candida albicans. Microbiology 1997, 143(Pt 2):303-311.

65. Shcherbo D, Merzlyak EM, Chepurnykh TV, Fradkov AF, Ermakova GV, Solovieva EA, Lukyanov KA, Bogdanova EA, Zaraisky AG, Lukyanov S, Chudakov DM: Bright far-red fluorescent protein for whole-body imaging. Nat Methods 2007, 4:741-746.

doi:10.1186/1752-0509-4-176

Cite this article as: Jungbluth et al:: Targeted protein depletion in Saccharomyces cerevisiae by activation of a bidirectional degron. BMC Systems Biology 2010 4:176.

\section{Submit your next manuscript to BioMed Central and take full advantage of:}

- Convenient online submission

- Thorough peer review

- No space constraints or color figure charges

- Immediate publication on acceptance

- Inclusion in PubMed, CAS, Scopus and Google Scholar

- Research which is freely available for redistribution 